\title{
PRÁTICAS DE ENSINO COM REALIDADE AUMENTADA POR MEIO DE SMARTPHONES: ESTUDO DE UMA PONTE FERROVIÁRIA
}

Maria Rafaela Bezerra de Macêdo Ribeiro-maria.ribeiro@coc.ufrj.br

Universidade Federal do Rio de Janeiro, Programa de Pós-Graduação em Engenharia Civil

Centro de Tecnologia - Ilha do Fundão

CEP 21941-909 - Rio de Janeiro - Rio de Janeiro

Nivea Gabriela Benevides de Albuquerque-nivea@ufpa.br

Universidade Federal do Pará, Faculdade de Engenharia Civil

Rua Augusto Corrêa s/n - Guamá

CEP 66075-110-Belém - Pará

Resumo: Nos últimos anos, notou-se a crescente demanda pela adoção de BIM em projetos de infraestrutura, de modo a auxiliar na gestão, seja no âmbito de concepção, construção ou manutenção das obras. Órgãos governamentais do setor já estimulam a sua aplicação pelas empresas. Nesse sentido, sabe-se também que, disciplinas base, tais como a análise estrutural desses projetos pode ser de difícil compreensão em um primeiro momento pelos alunos nas faculdades de engenharia. O presente trabalho tem por apresentar a aplicação de realidade aumentada do projeto de uma ponte ferroviária treliçada, existente no Estado do Maranhão, para a sua demonstração será utilizado um programa comercial disponível em aparelhos móveis, de modo a aumentar a interatividade do usuário com o modelo, bem como a flexibilidade de poder aplicá-lo em diferentes ambientes.

Palavras-chave: Realidade Aumentada. Pontes Ferroviárias. BIM.

\section{INTRODUÇÃO}

Ao pensar nos grandes avanços tecnológicos dos últimos anos, percebe-se cada vez mais a necessidade de agregar ao ensino de engenharia, os benefícios advindos por tais tecnologias disponíveis no mercado. É evidente se pensar em práticas pedagógicas de forma que essas tecnologias possam ser inseridas de forma efetiva no dia a dia de sala de aula, de forma que fique alinhada com os objetivos didáticos.

O uso de computação gráfica em dispositivos móveis, tais como tablets e smartphones, é algo possível e eficaz no sentido que pode instigar os alunos a desenvolverem os seus próprios modelos estruturais. Atualmente, nota-se cada vez mais o aumento da capacidade de processamento gráfico desses dispositivos, ampliando dessa forma o acesso as tecnologias de Realidade Aumentada (RA) e Realidade Virtual (RV), que já são bem aplicáveis e conhecidos no mercado de entretenimento. Sabe-se que, essas ferramentas podem ser de grande valia para os alunos que possuem dificuldades de visualizar e compreender o comportamento e disposição dos elementos estruturais em termos tridimensionais.

Nesse sentido, sabendo-se das potencialidades das tecnologias digitais atuais, incluindo a comunicação, processamento numérico e a computação gráfica, o grande desafio é aliar os dispositivos (computador, celular, tablet, etc), ao processo ensino aprendizagem como objetos educacionais capazes de aperfeiçoar e tornar mais dinâmica as aulas. É preciso buscar meios de usufruir dessas ferramentas e criar métodos de apresentação de conteúdo que possam 
contribuir para assimilação de conceitos e na potencialização dos métodos de estudo dos alunos e das metodologias de ensino. (MACÊDO; SILVA; BURIOL, 2016).

O trabalho a ser desenvolvido corresponde a um projeto iniciado em 2017 na UFPa, na faculdade de Engenharia de Ferroviária e Logística, no qual buscam-se novas formas alternativas de ensino, adotando a realidade aumentada para o desenvolvimento de modelos realísticos, que inicialmente foram desenvolvidas para pontes ferroviárias e material rodante, expandindo-se posteriormente para o estudo de componentes da ferrovia, seja no âmbito da via permanente (com seus elementos macro de constituição tais como lastro, dormentes e trilhos), bem como projetos de AMV's (Aparelho de Mudança de Via) e Maglev. Para isso foram utilizados os programas comerciais Unity $3 \mathrm{D} \AA$ para a aplicação da RA e o Vuforia ${ }^{\circledR}$ para a criação do banco de dados, onde até o presente momento são modelos interativos pela câmera do computador. No sentido de tentar expandir para plataformas móveis será adotado o programa comercial Augin ${ }^{\circledR}$ de realidade aumentada, disponível em dispositivos móveis, tendo como aplicação inicial uma ponte ferroviária treliçada que existe no Estado do Maranhão.

\section{REVISÃO BIBLIOGRÁFICA}

\subsection{Origem da Realidade Virtual e Aumentada}

Segundo Carmigniani et al. (2011), a história cronológica da evolução desta ciência deuse com a realidade virtual na década de 30, quando Edward Link inventou o Link Trainer, o primeiro exemplo de um simulador de voo comercial. Na década de 50, começou a surgir o conceito de realidade aumentada, quando Morton Heilig, diretor de fotografia, viu o cinema como um elemento que poderia incorporar o espectador como ator principal do filme. Em 1962, Heilig concebeu um protótipo da sua ideia, o Sensorama. Em 1975, Myron Krueger criou o termo de realidade aumentada. Azuma (1997) escreve o primeiro artigo acerca do estado da arte, definindo cientificamente o significado de RA e nesse mesmo ano é criado o primeiro protótipo de RA para um dispositivo móvel com recurso à tecnologia de GPS.

No âmbito da quarta revolução industrial, ou simplesmente indústria 4.0, são apresentados novos desafios tanto para a indústria como no quesito ensino e aprendizagem, sendo a RA uma das tecnologias a serem incorporadas na indústria 4.0, segundo a CNI (2017).

No setor ferroviário a RA apresenta-se altamente promissora, em vista da necessidade de profissionalização e capacitação de pessoas para o desenvolvimento logístico bem como a expansão da malha brasileira.

Figura 1 - Usuário interagindo com modelo RA de um truque ferroviário.

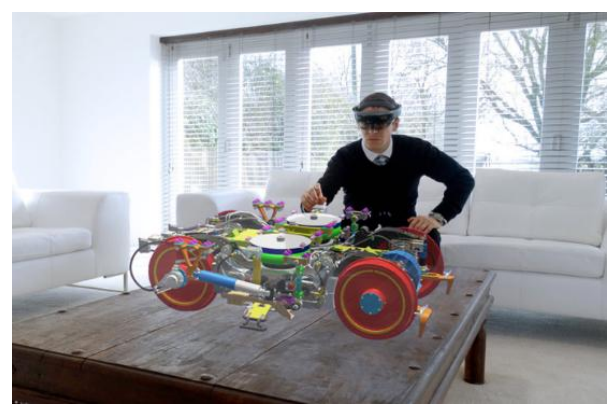

Fonte: Pauley.co.uk 
Segundo Milgram et al. (1994) a RA está contida dentro de um subconjunto da realidade misturada, localizada dentro de um continuum de virtualidade, como é apresentada na figura 2 a seguir:

Figura 2 - Subconjuntos da Realidade Misturada.

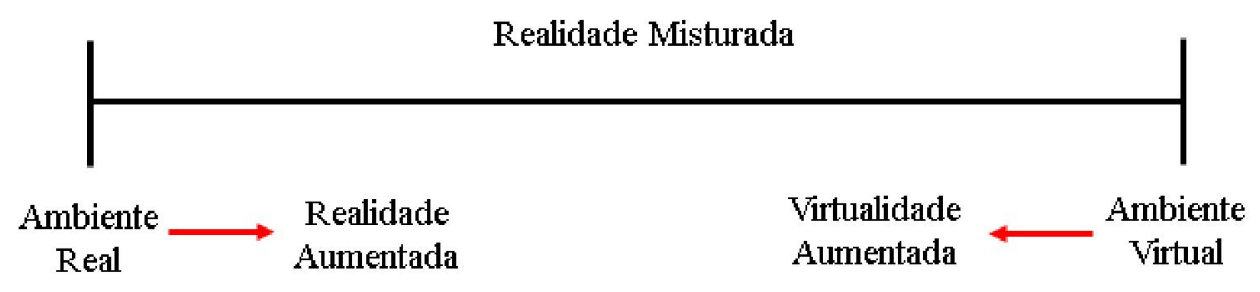

Fonte: Milgram et al. (1994), adaptada.

Nota-se que, a RA está mais próxima do ambiente real, de modo que, os objetos virtuais gerados nesse ambiente são para enriquecer e melhorar a nossa interatividade com a nossa realidade. O objetivo não é fornecer uma experiência completamente imersiva, como ocorre na realidade virtual, mas sim melhorar a interação do usuário com o mundo real. Dessa forma, não é necessário tanto poder de processamento, pois não é preciso simular um mundo totalmente novo, como também não é preciso nenhum dispositivo especial, atualmente com um smartphone já é possível transferir uma aplicação que faça uso desta tecnologia, tal como o jogo que ficou famoso por trazer à tona a popularização da RA na área de entretenimento, o Pokémon GO® em 2016. Na figura 3 é apresentada a interação do usuário com este jogo.

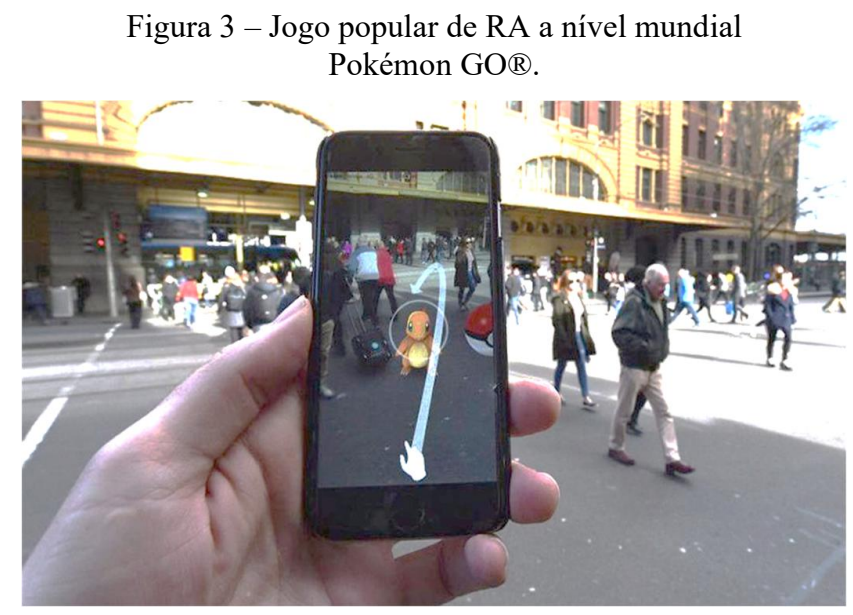

Fonte: Palazuelos, 2016.

\subsection{Desenvolvimento de Projetos BIM}

Cada empreendimento engloba colaboradores das mais diversas áreas, sejam os projetistas, construtores, operários, gestores, e todos precisam ter um entendimento global, desde a sua concepção, construção e manutenção de modo a otimizar os custos e por conseguinte, possíveis falhas. Nesse sentido, o uso do BIM (Building Information Modeling), ou seja, Modelagem da Informação da Construção, vem trazer de forma visual todas as informações pertinentes do projeto.

Em 2018, foi publicado o decreto $n^{\circ}$ 9337, que determina a difusão do BIM em obras de grande porte pelo país, designada como Estratégia BIM-BR, onde o DNIT é o principal órgão 
(C) COBENGE 2020

"Os desafios para formar hoje o engenheiro do amanhã"
$\mathrm{Ol} \mathrm{a} \mathrm{O} 3$ de dezembro Evento On-line

responsável, visando coordenar a estruturação do setor público para a adoção, de modo a favorecer as condições das empresas para o investimento público e privado, para a geração de parâmetros de compras e contratações, normas técnicas, bem como novas tecnologias para o seu desenvolvimento. Na figura 4 é apresentado o exemplo de projeto BIM de duplicação de uma rodovia em São Paulo.

Figura 4 - Modelo BIM de projetos em infraestrutura.

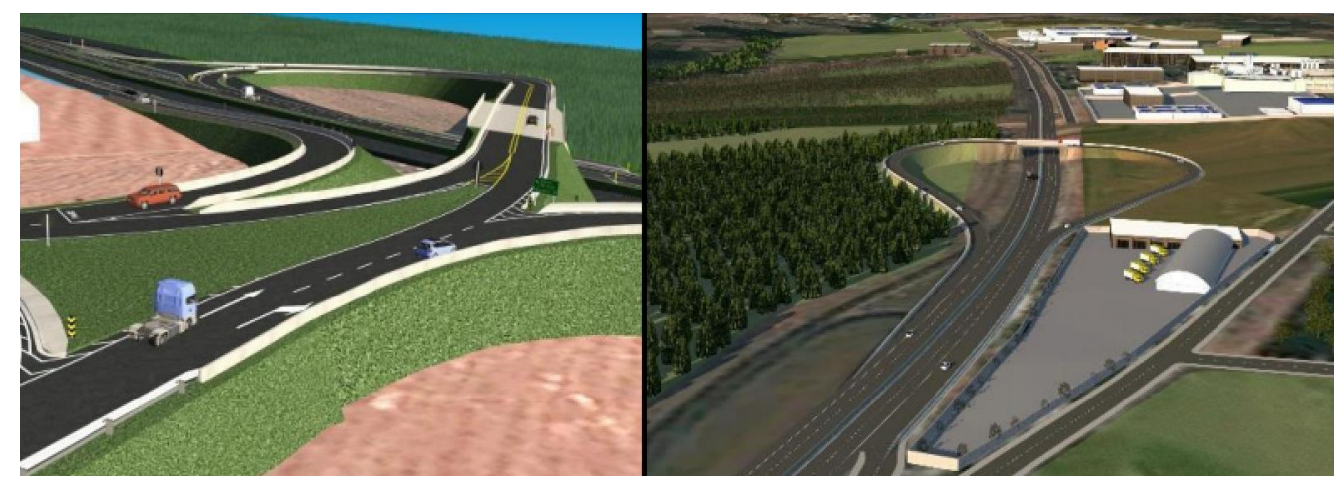

Fonte: Cursino, 2017.

\subsection{Modelos RA na Engenharia}

Trabalhos recentes já são realizados em RA com enfoque na engenharia, em especial na área civil. Almeida et al. (2018) aplicaram RA em smartphones, com enfoque ao ensino de instalações elétricas. Scortegagna e Gonçalves (2018) utilizaram a RA na disciplina de desenho técnico, o que tornou a disciplina mais dinâmica e participativa entre os alunos. Parcianello e Lemos II (2019), realizaram um trabalho semelhante ao apresentado anteriormente, mas foi realizado por meio de web-cam. Júnior et al. (2020), aplicaram a RA ao ensino de estruturas de concreto armado, em conjunto a impressão 3D. Ribeiro e Albuquerque (2019), desenvolveram um trabalho de forma a aplicar RA em pontes ferroviárias, na qual foi desenvolvida uma cartilha de marcadores, onde alguns destes marcadores já eram as próprias plantas de projeto, que eram reconhecidos pela câmera de um computador. Na figura 5 é mostrado a visualização do modelo RA da ponte pelo computador.

Figura 5 - Modelo RA da ponte completa.

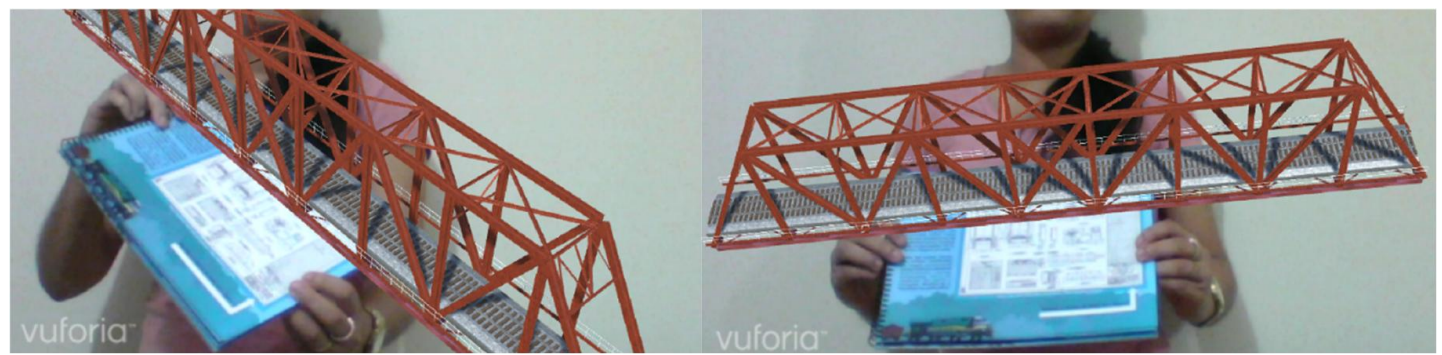

Fonte: Ribeiro e Albuquerque, 2019. 


\section{METODOLOGIA}

\subsection{Programas Comerciais}

- Vuforia e Unity 3D ${ }^{\circledR}$

O software Vuforia, é um programa de armazenagem de marcadores, no qual possui como vantagem a utilização de imagens diversas com reconhecimento $2 \mathrm{D}$ para de realidade aumentada, além dos $Q R C o d e s$ já conhecidos, no qual é realizado o reconhecimento de pontos de captura pela câmera, possuindo plugin para o software Unity $3 \mathrm{D} \AA$, que é uma plataforma que ficou popular inicialmente para o desenvolvimento de jogos, mas que já está se expandindo para a área da engenharia e arquitetura, sendo versátil para várias linguagens computacionais, tais como C\#, Javacript, dentre outros, para o desenvolvimento VR e AR. Além disso, possui extensões para criação de aplicativos em sistemas Android e iOS.

\section{- Augin ${ }^{\circledR}$}

É um programa comercial gratuito de realidade aumentada desenvolvida no Brasil, e se tornou disponível para as plataformas Android e iOS a partir de 2019, e também para aparelhos desenvolvidos a partir de 2017, com o intuito de desenvolvimento e apresentação de projetos de arquitetura e engenharia de modo a diversificar e proporcionar ao usuário uma maior interação com o meio ao qual está inserido, tornando mais dinâmico o processo. Os plugins já podem ser adicionados a vários outros programas, sendo os principais, SketchUP®, Revit $\AA$ e TQS $\AA$.

\subsection{Estudo de Caso}

O estudo de caso, baseia-se em uma OAE localizada no Estado do Maranhão, existente de uma ferrovia dedicada principalmente ao transporte Heavy Haul. A ponte em questão é treliçada e possui um vão de 70 metros. Na figura 6 é mostrada a vista lateral da ponte na qual será aplicada a metodologia.

Figura 6 - Ponte ferroviária real.

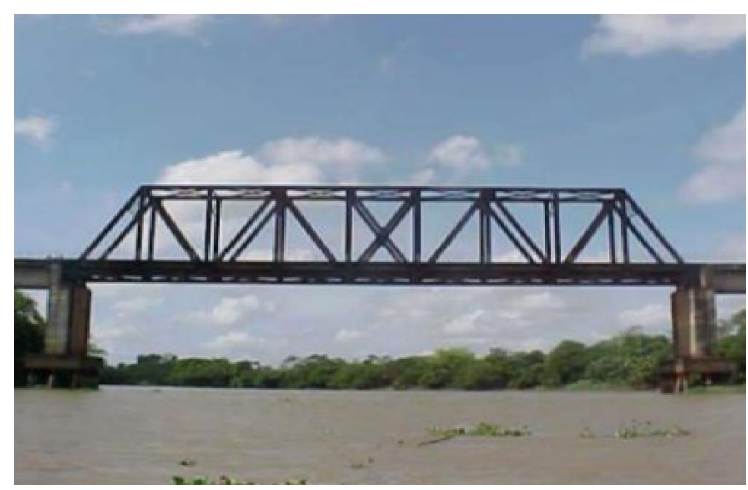

Fonte: Lobato et al., 2009.

\subsection{Desenvolvimento do Modelo}

A modelagem da ponte foi realizada com base nas plantas originais do projeto. Quanto a superestrutura da via permanente, esta foi considerada com uma laje de concreto onde foram dispostos, o lastro, dormentes e o trilho do tipo TR-68. As ferramentas gráficas utilizadas para a sua concepção inicialmente foi o software Autocad ${ }^{\circledR}$, no qual posteriormente foi escolhido 
Figura 9 - Manipulação do modelo da ponte.

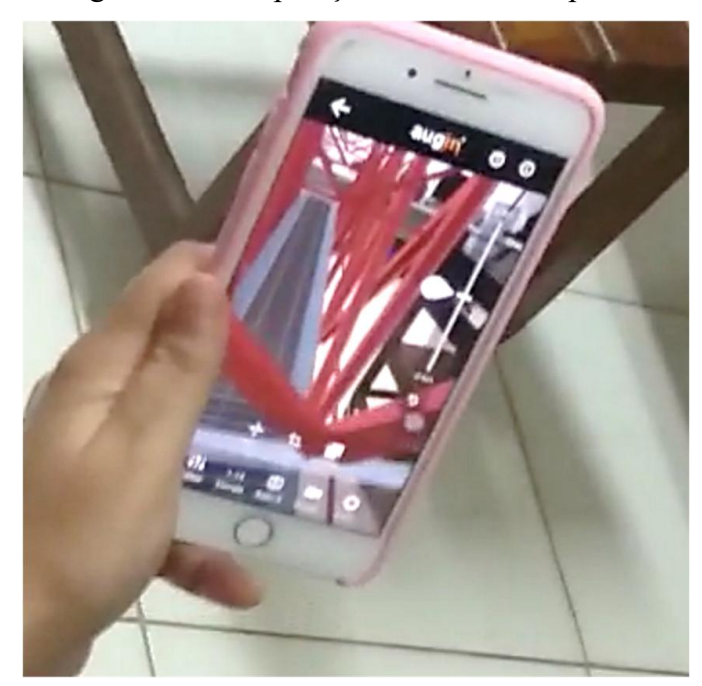

Fonte: Autoras.

É possível também fazer registros fotográficos bem como em vídeo da utilização do modelo. Na figura 10 é mostrado também que o modelo RA é bem reconhecido em diversas superfícies que o usuário deseje aplicá-lo.

Figura 10 - Interação do modelo RA com o ambiente real.

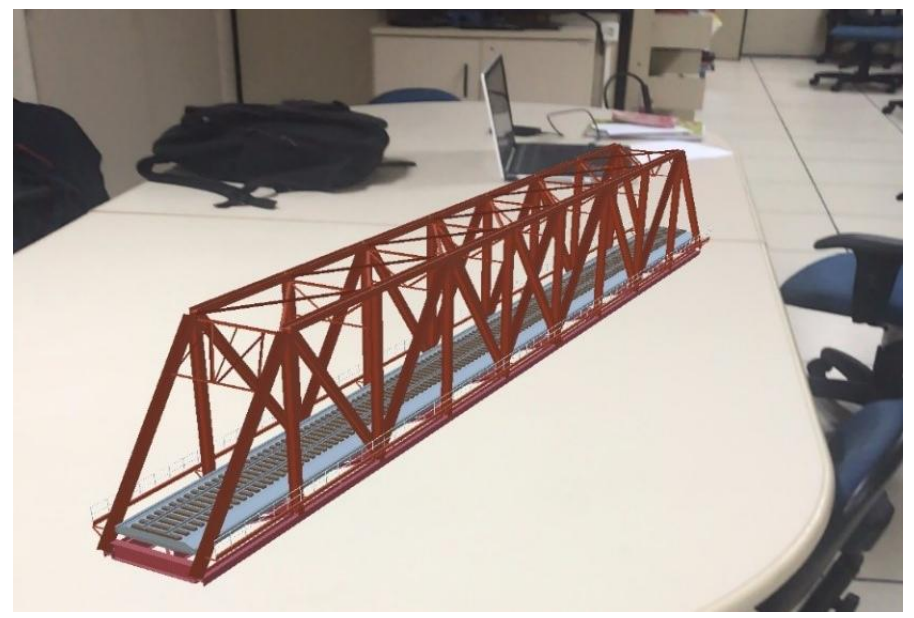

Fonte: Autoras.

Os alunos se sentem mais instigados e interagem mais no processo ensino-aprendizagem. Por se tratar de uma metodologia ativa, ou seja, centrada no aluno visando engajá-lo ao contexto, observou-se ainda uma aprimoração nas habilidades de compreensão, aplicação, síntese e avaliação do objeto em estudo, o que potencializou a investigação da ponte. É importante frisar ainda que a mente aberta dos jovens deu ensejo a uma boa receptividade, permitindo difundir o uso dessa oportuna tecnologia. A apresentação dessa proposta de modo a estender para aplicação rápida em smartphone foi dada para o grupo PET de Engenharia 
Civil da UFPa, sendo bem aceita pelos mesmos, estimulando o aprendizado também da programação, como é mostrado na figura 11 .

Figura 11 - Alunos no processo de aprendizagem da realidade aumentada.

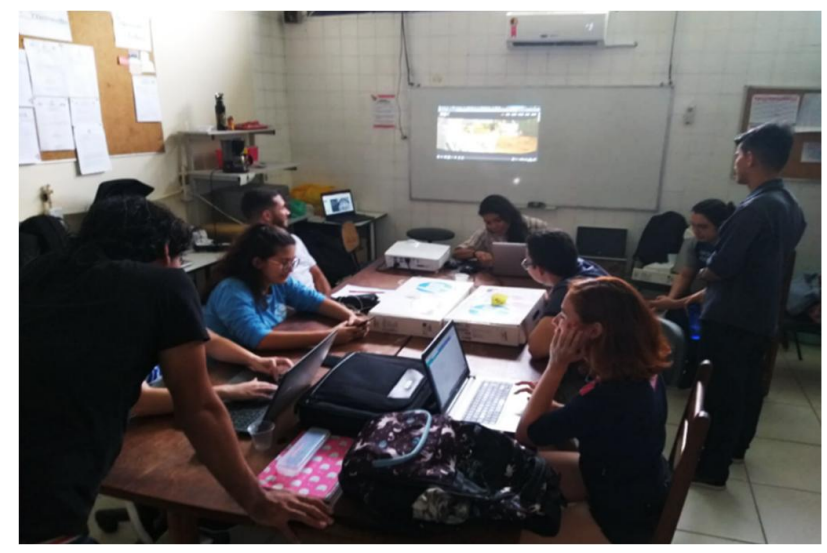

Fonte: Autoras.

\section{CONSIDERAÇÕES FINAIS}

A realidade aumentada mostrou-se uma rica ferramenta para o ensino de estruturas, com potencial para ser explorada em outras áreas. $\mathrm{O}$ uso da RA em smartphones torna o processo ainda mais flexível e acessível, podendo ser aplicado em diferentes ambientes. Notou-se que a metodologia instiga a vontade e a curiosidade dos estudantes, fazendo com que aprendam de forma mais ativa e prática algo que até então era visto de forma abstrata por eles nas aulas relacionadas a estruturas. Nesse sentido, a RA pode tornar as aulas mais dinâmicas e interativas, incentivando assim, com que os alunos desenvolvam os seus próprios modelos, para a apresentação de seus projetos.

\section{Agradecimentos}

As autoras agradecem à Pró-Reitoria de Ensino de Graduação da Universidade Federal do Pará (PROEG/UFPA) pelo fomento ao desenvolvimento do projeto PGRAD1800470101326, vinculado ao Programa de Apoio à Qualificação do Ensino de Graduação (PGRAD), Subprograma de Apoio à Infraestrutura de Laboratórios de Ensino de Graduação LABINFRA/2018.

\section{REFERÊNCIAS}

ALMEIDA, Bruno. R.; GUIMARAES, Jéssica S.; BRITO, Welligton. A.; MAGALHAES, Francisco. R. P.; OLIVEIRA, Átila. G. Realidade aumentada aplicada as práticas laboratoriais nos cursos de engenharia. In: XLVI Congresso Brasileiro de Educação em Engenharia e $1^{\circ}$ Simpósio Internacional de Educação em Engenharia, 2018, Salvador. Anais. Salvador, 2018.

ATOS DO PODER EXECUTIVO. Decreto nº 9377. Distrito Federal, 2018.

AUGIN. Disponível em: https://augin.app/plugins/. Acesso em: 11 abr. 2020. 
"Os desafios para formar hoje o engenheiro do amanhã"

AZUMA, Ronald. T. A Survey of Augmented Reality. Teleoperators and Virtual Environments, Malibu, v. 6, n. 4, p. 355-385, 1997.

CARMIGNIANI, Julie.; FURHT, Borko.; ANISETTI, Marco.; CERAVOLO, Paolo.; DAMIANI, Ernesto.; IVKOVIC, Misa. Augmented reality technologies, systems and applications. Multimedia Tools and Applications, v. 51, n. 1, p. 341-377, 2011.

CNI (Confederação Nacional da Indústria). Oportunidades para a indústria 4.0 - Aspectos da demanda e oferta no Brasil. Brasília, p. 58, 2017.

CURSINO, Pedro. L. S. Tomada de decisões em projetos de infraestrutura. In: $1^{\text {a }}$ Semana do Planejamento, 2017, Brasília, Anais. Brasília, 2017.

JÚNIOR, Amauri. S. R.; GOMES, Gustavo. J. C.; BERTEGES, Luiz. F. C.; PEREIRA, Cristiane. S. C.; CARVALHO, Carlos. V. A. Um material potencialmente significativo para o ensino da engenharia civil utilizando impressora 3D e realidade aumentada: uma experiência com alunos do ensino médio e do ensino superior. Brazilian Journal of Development, Curitiba, 7 mar. 2020. p. 10855.

LOBATO, Selma Lídia Azevedo et al. Análise modal experimental e computacional de uma ponte metálica ferroviária. Construção Metálica, São Paulo, v.1, n.92, p. 24-28, 2009.

MACÊDO, Alex. C; SILVA, João. A; BURIOL, Tiago. M. Usando smartphone e realidade aumentada para estudar geometria espacial. Revista Renote, Porto Alegre, v. 14, n. 2, p. 1-10, 2016.

MILGRAM, Paul; TAKEMURA, Haruo; UTSUMI, Akira; KISHINO, Fumio. Augmented Reality: A class of displays on the reality-virtuality continuum. Telemanipulator and Telepresence Technologies, v. 2351, n. 1, p. 282 - 292, 1994.

PALAZUELOS, F. "Pokemon Go", os motivos que alcançaram o fenômeno da realidade aumentada. Disponível em: https://brasil.elpais.com/brasil/2016/07/12/tecnologia/1468336791_763102.html. Acesso em: 11 abr. 2020.

PARCIANELLO, Yussef; LEMOS II, Dalton Luiz. Utilização de realidade aumentada buscando maior engajamento dos alunos no ensino de desenho técnico e desenho assistido por computador. Engenharia no século XXI. 1ª. ed. Belo Horizonte: Ed. Poisson, 2019. p. 54 62 .

PAULEY, B. Augmented and virtual reality are a key driver in delivering the digital Railway. Disponível em: https://www.pauley.co.uk/blog/augmented-and-virtual-reality-are-akey-driver-in-delivering-the-digital-railway/. Acesso em: 11 abr. 2020.

RIBEIRO, Maria Rafaela Bezerra de Macêdo; ALBUQUERQUE, Nívea Gabriela Benevides de. Aplicação de realidade aumentada para projetos de pontes ferroviárias. Engenharia no século XXI. $1^{\text {a }}$. ed. Belo Horizonte: Ed. Poisson, 2019. p. 179-190. 
"Os desafios para formar hoje o engenheiro do amanhã"

SCORTEGAGNA, Luan. F.; GONÇALVES, Flávia. A. Utilização de realidade aumentada na otimização do ensino-aprendizagem de desenho técnico na engenharia. In: XLVI Congresso Brasileiro de Educação em Engenharia e $1^{\mathrm{o}}$ Simpósio Internacional de Educação em Engenharia, 2018, Salvador. Anais. Salvador, 2018.

UNITY 3D. Disponível em: https://unity.com/pt. Acesso em: 11 abr. 2020.

VUFORIA. Disponível em: https://developer.vuforia.com/. Acesso em: 11 abr. 2020.

\title{
TEACHING PRACTICES WITH AUGMENTED REALITY THROUGH SMARTPHONES: STUDY OF A RAILWAY BRIDGE
}

\begin{abstract}
In recent years, there has been a growing demand for the adoption of BIM in infrastructure projects, in order to assist in management, whether in the scope of design, construction or maintenance of works. Government agencies in the sector are already stimulating their application by companies. In this sense, it is also known that basic disciplines, such as the structural analysis of these projects can be difficult to understand at first by students in engineering faculties. The present work has to present the application of augmented reality of the project of a latticed railway bridge, existing in the State of Maranhão, as well as some of its components adopting for its demonstration a commercial program available in mobile devices in order to increase the interactivity of the with the model, as well as the flexibility to be able to apply it in different environments.
\end{abstract}

Keywords: Augmented Reality, Railway Bridge, BIM. 